\title{
Analysis and modeling of time-course gene-expression profiles from nanomaterial-exposed primary human epidermal keratinocytes
}

\author{
Amin Zollanvari ${ }^{1}$, Mary Jane Cunningham ${ }^{2,5}$, Ulisses Braga-Neto*1 \\ and Edward R Dougherty ${ }^{1,3,4}$
}

\author{
Address: ${ }^{1}$ Department of Electrical and Computer Engineering, Texas A\&M University, College Station, TX, USA, ${ }^{2}$ Nanomics Biosciences, Cary, \\ NC, USA, ${ }^{3}$ Department of Pathology, University of Texas MD Anderson Cancer Center, Houston, TX, USA, ${ }^{4}$ Computational Biology Division, \\ Translational Genomics Research Institute, Phoenix, AZ, USA and ${ }^{5}$ Previous affiliation: The Houston Advanced Research Center, \\ The Woodlands, TX, USA \\ E-mail: Amin Zollanvari - amin_zoll@neo.tamu.edu; Mary Jane Cunningham - mjcunningham@nanomicsbio.com; \\ Ulisses Braga-Neto* - ulisses@ece.tamu.edu; Edward R Dougherty - edward@ece.tamu.edu \\ *Corresponding author
}

from Sixth Annual MCBIOS Conference. Transformational Bioinformatics: Delivering Value from Genomes Starkville, MS, USA 20-2I February 2009

Published: 08 October 2009

BMC Bioinformatics 2009, I0(Supp| II):SI0 doi: I0.II86/I47|-2105-I0-SII-SI0

This article is available from: http://www.biomedcentral.com/|47|-2/05/I0/SII/SI0

(C) 2009 Zollanvari et al; licensee BioMed Central Ltd.

This is an open access article distributed under the terms of the Creative Commons Attribution License (http://creativecommons.org/licenses/by/2.0), which permits unrestricted use, distribution, and reproduction in any medium, provided the original work is properly cited.

\begin{abstract}
Background: Nanomaterials are being manufactured on a commercial scale for use in medical, diagnostic, energy, component and communications industries. However, concerns over the safety of engineered nanomaterials have surfaced. Humans can be exposed to nanomaterials in different ways such as inhalation or exposure through the integumentary system.

Results: The interactions of engineered nanomaterials with primary human cells was investigated, using a systems biology approach combining gene expression microarray profiling with dynamic experimental parameters. In this experiment, primary human epidermal keratinocytes cells were exposed to several low-micron to nano-scale materials, and gene expression was profiled over both time and dose to compile a comprehensive picture of nanomaterial-cellular interactions. Very few gene-expression studies so far have dealt with both time and dose response simultaneously. Here, we propose different approaches to this kind of analysis. First, we used heat maps and multidimensional scaling (MDS) plots to visualize the dose response of nanomaterials over time. Then, in order to find out the most common patterns in gene-expression profiles, we used self-organizing maps (SOM) combined with two different criteria to determine the number of clusters. The consistency of SOM results is discussed in context of the information derived from the MDS plots. Finally, in order to identify the genes that have significantly different responses among different levels of dose of each treatment while accounting for the effect of time at the same time, we used a two-way ANOVA model, in connection with Tukey's additivity test and the Box-Cox
\end{abstract}


transformation. The results are discussed in the context of the cellular responses of engineered nanomaterials.

Conclusion: The analysis presented here lead to interesting and complementary conclusions about the response across time of human epidermal keratinocytes after exposure to nanomaterials. For example, we observed that gene expression for most treatments become closer to the expression of the baseline cultures as time proceeds. The genes found to be differentiallyexpressed are involved in a number of cellular processes, including regulation of transcription and translation, protein localization, transport, cell cycle progression, cell migration, cytoskeletal reorganization, signal transduction, and development.

\section{Background}

Nanomaterials are being manufactured on a commercial scale for use in medical, diagnostic, energy, component and communications industries [1,2]. Engineered nanomaterials range considerably in their physicochemical properties making them more desirable than their microand macro-counterparts due to, for example, their increased surface area, tensile strength, tunability, etc. [3]. From limited early reports, concerns over the safety of engineered nanomaterials have surfaced [4,5]. Humans can be exposed to nanomaterials in different ways such as inhalation or exposure through the integumentary system. However, the skin is a unique organ in the body not only because it gives the body such a large surface area for exposure but also because of the avascular property of epidermis, in which particles can reside without being removed by phagocytosis [6].

Gene expression microarrays have become a tool to investigate the interactions of biological systems by observing the simultaneous activities of tens of thousands of genes. Over recent years, this tool has been applied to toxicology forming a new discipline, toxicogenomics $[7,8]$. Microarrays have most recently been a tool used by pharmaceutical drug discovery and development to screen for efficacy and adverse effects thereby prioritizing drug candidates and redeveloping ones which show off-target toxicities [9-11].

The approach described here combines a global screening technology, gene expression microarray profiling, with systems biology, to investigate the interactions of engineered nanomaterials with primary human cells. The biological and cellular system is perturbed and reiteratively sampled over both time and dose to compile a more comprehensive picture of nanomaterial-cellular interactions. From over 100 papers which were reviewed by the authors in [12], only 3 papers have dealt with the effect of concentration plus time while the remaining papers dealt only with the parameter of time. Initial studies that were published previously focused on reporting significantly-expressed genes and using clustering methods to identify similarities and differences between expression profiles [13-15]. In addition, the study cited in [12] was the only previously combined study investigating time-course and dosage-effect simultaneously, while the initial 3 cited studies investigated time-effect and dosage effect separately.

In the present study, we propose different approaches to this kind of analysis. We considered the gene expression of primary human epidermal keratinocytes, under exposure to the following low-micron to nano-scale materials: carbonyl iron (FC), carbon black (CB) silica $\left(\mathrm{SiO}_{2}\right)$ and single-walled carbon nanotubes (SWNT), at noncytotoxic and cytotoxic doses for each. The nanomaterials used, except for the carbonyl iron (FC) and SWNT, are not intended for medical use. These materials are currently being used in construction materials, consumer goods, and communications and IT applications. The FC nanoparticles were used as a negative control compound and have been approved for use by the FDA as a pharmaceutical carrier formulation. The single-walled carbon nanotubes are being developed for medical applications (e.g. drug carriers or medical imaging compounds) only after being functionalized with other components. We remark here that the goal of the experimental design was not to study particle size or penetration effects. It was to study whether there was an overall interaction with the nanomaterials. In particular, the cytotoxic dose (i.e. high dose) used with the carbonyl iron was due to its toxic effect of an overload of iron on the cells.

In the approach discussed here, we first used heat maps and multi-dimensional scaling (MDS) plots to visualize the dose response of nanomaterials over time. Then, in order to find out the most common patterns in geneexpression profiles, we used self-organizing maps (SOM) combined with two different criteria to determine the number of clusters. The consistency of the SOM results is discussed in context of the results from the MDS plots. Finally, in order to identify the genes that have significantly different responses among the different 
dose levels while simultaneously accounting for the effect of time, we have used a two-way ANOVA model, in connection with Tukey's additivity test and the Box-Cox transformation. These analysis results are discussed in the context of the biological and cellular interactions of the engineered nanomaterials.

\section{Methods}

\section{Biological experimental design}

Primary human epidermal keratinocytes (HEK, Cascade Biologics) were cultured with serum-free defined media at $5 \% \mathrm{CO}_{2}$ and $37^{\circ} \mathrm{C}$. The HEK were exposed to the following low-micron to nano-scale materials: carbonyl iron (FC), carbon black (CB) silica $\left(\mathrm{SiO}_{2}\right)$ and singlewalled carbon nanotubes (SWNT). FC was supplied by ISP Technologies with a mean particle size of $5.8 \mu \mathrm{m}, \mathrm{CB}$ was supplied by Degussa with a mean particle size of $17 \mathrm{~nm}$, and $\mathrm{SiO}_{2}$ was supplied by US Silica with a mean particle size of $1.6 \mu \mathrm{m}$. SWNT was prepared by SouthWest Nanotechnologies with a mean diameter of $0.8 \mathrm{~nm}$. The purity of SWNT was checked, and it was found that all heavy metal contamination was very low, less than $1 \%$. The nanotubes were acid-purified from the raw manufactured intermediate. The average length of the SWNT nanotubes was $960 \mathrm{~nm}$; this is the only nanomaterial among the ones considered here that is not spherical. From previous viability assays $[13,15]$, two different doses were selected for the exposure of each nanomaterial - a noncytotoxic dose and a cytotoxic dose. The cytotoxic dose was extrapolated from the viability curve of each substance where $50 \%$ of the cells were still viable. The noncytotoxic and cytotoxic doses for each nanomaterial were found to be, respectively, 0.03 and 1 (FC), 0.01 and $0.5(\mathrm{CB}), 0.1$ and $1\left(\mathrm{SiO}_{2}\right)$, and 0.001 and 1 (SWNT), all concentrations being expressed in $\mathrm{mg} / \mathrm{ml}$. The cells were exposed to $\mathrm{FC}, \mathrm{CB}$, $\mathrm{SiO}_{2}$, and SWNT and harvested at 2, 4, 6, 8, 12, 18, and 24 hours after exposure. Cells cultured under the same conditions and not exposed to any of the nanomaterials were harvested at the same time points for a timematched control baseline.

The cells were trysinized and cell counts taken. The cells were then collected by centrifugation, snap frozen in liquid nitrogen, and stored at $-80^{\circ} \mathrm{C}$. The frozen pellets were shipped to GenUs Biosystems for isolation of the total RNA and processing of the gene expression microarrays. Total RNA was isolated from the cell pellets, reverse-transcribed to biotinylated cRNAs and hybridized onto $10 \mathrm{~K}$ human gene expression microarrays (GE Healthcare). The corresponding cRNA for each biological sample was hybridized to triplicate microarrays. The arrays were rinsed, dried, scanned and image analyzed and the flat files returned to the Houston Advanced Research Center.

\section{Data analysis and preprocessing}

The microarray flat files contained the quantitative expression values for all probes (positive controls, negative controls, fiducial, and discovery) and all discovery probe values were assessed with a quality flag. Only those probes that have "DISCOVERY" and "G" (Good-quality) tags across all time points and dose levels of all treatments were considered for further analysis to guarantee maximal reproducibility of the results. By using this criterion, the number of genes was reduced from 10,458 to 2,370 . Then the average of the three replicate measurements was considered as the actual expression level of each gene at that time in its corresponding treatment.

\section{Heatmaps}

The heatmaps are visualizations of hierarchical clustering [16] using "average" linkage and Pearson Correlation as the distance metric between expression levels of each gene after taking the log ratio of the data. It is worthy to mention that Pearson correlation is in fact the normalized correlation between values of two random variables that have had their mean subtracted from them. The dendrogram added to the left side of each heat map is obtained by Pearson correlation. The heatmaps were drawn by first subtracting the mean of each row from each data cell and then normalizing each row to obtain a variance of 1 . Note that normalization was performed after taking the log ratio of the expression of each gene between the given treatment and the control baseline. Heatmaps obtained in this way reveal the underlying shape of expression pattern of genes better and are less affected by some large values which may be the result of noise. For more information on applying hierarchical clustering to gene expression patterns, see [16] and [17] and the references mentioned there.

\section{Multi-dimensional Scaling (MDS)}

Multidimensional Scaling (MDS) is a method to project high dimensional data onto a lower dimension while maintaining the approximate distances between data points [21]. The accuracy of the representation is measured by the "stress." A stress of $10 \%$ is usually considered good.

First, the data are collected by taking the log of the expression values of genes in each treatment and the baseline. Then at each time point, a matrix of distances between the gene-expression profiles corresponding to each treatment and the baseline is created, where the 
distance is calculated as 1 - the Pearson correlation between the profiles. Finally, MDS is performed based on this matrix, in order to obtain a low-dimensional representation of the gene-expression profiles and the distance among them.

\section{Self-Organizing Maps (SOM)}

SOM [15] is a powerful method for clustering. After lognormalizing the data as explained above, this method was employed in two ways.

First, the underlying hidden number of clusters of the data was estimated using two different criteria the silhouette criterion [18] and the CLEST criterion [19]. In [19], it has been shown that the CLEST criterion is the stronger tool to find number of clusters. However, from the comparison of the CLEST and the silhouette criteria in [19], it is inferred that whenever the true number of clusters has been equal to 2, the silhouette criterion is the same as the CLEST or has done a better job than the CLEST criterion, while for greater number of clusters, the CLEST performs better. Therefore, the criterion that we have used to determine the number of clusters is a combination of the two criteria. First, the silhouette criterion is implemented to determine the number of clusters. If the number of clusters for any treatment is equal to 2, and if the silhouette width for this number of clusters is fairly close to 1 (e.g. greater than 0.6 ) then we assigned 2 as the optimal number of clusters. Otherwise, the CLEST criterion is implemented to determine the number of clusters.

In the second way of implementing SOM, larger numbers of clusters than the previous approach were tried, as a means of obtaining clusters that show tighter expression patterns than in the first approach.

\section{ANOVA model}

A two-way ANOVA model was used to identify genes for which there are significant differences in the response to the various dose levels, and have them ranked by the $p$-values of dose main effect. The general procedure was as follows:

0 - For each gene, compile the data in a twodimensional array consisting of the expression values from the three levels of dose (untreated, low and high) and the eight time points. Use Tukey's additivity test for two-way ANOVA (one observation per cell) to see if there is interaction between time and dose for the given gene. If there is no interaction go to step 2.

1 - If there is significant interaction between these two factors, try to remove the interaction by using the
Box-Cox transformation [20]. The removal of interaction can be tested by Tukey's additivity test for transformed values corresponding to each parameter of the Box-Cox transformation. We have chosen this parameter to vary between -2 to 2 with 0.1 spacing. If the interaction is removed, use these as the new data for the given gene. If the interaction could not be removed, set the p-value of dose main effect to NA and go to step 0 to start the process for the next gene. 2 - Use two-way ANOVA to find whether there are significant differences among the levels of the dose and time factor. If there is not any significant difference on any factor, go to step 4 .

3 - Use Tukey's HSD post-hoc test to determine between which levels of either factor there are significant differences and record them. Since Tukey's HSD test is conservative, in rare cases the test cannot find any significant difference between levels.

4 - Save the p-value for the difference in dose main effect for this gene and go to step 0 to start the process for the next gene.

At the end of this process, sort all genes with respect to p-values on dose main effect. The lower the p-value is for a gene, the stronger the evidence is that there are significant differences in response among the various levels of dose for that gene.

\section{Results}

\section{A. Heatmap plots}

For the heatmaps, we consider the log ratio between the gene expression in one of the treatment time-courses and the corresponding gene expression in the baseline time-course, for each gene and each time point, and plot the log ratio for each gene (rows) versus time point (columns). There are 8 heatmaps, corresponding to each of the 8 treatments (including low and high dose), which are displayed in Figures 1 and 2. In these plots, the closer the color of a cell is to white (positive log ratio, up-regulation) and red (negative log ratio, downregulation), the more difference there is between the expression value of that gene between the given treatment and the baseline at the particular time point; by contrast, orange indicates an absence of differential expression with respect to the baseline. Considering, for instance, the $\mathrm{SiO}_{2} \mathrm{LD}$ (low dose) heatmap, we can see that differential expression tends to occur early in the time course (between 0 and $4 \mathrm{hr}$ ) and tends to disappear with time. It can also be observed in this heatmap that the genes fall in basically two clusters with respect to their time-course expression: a majority of genes that are up-regulated early, and a minority that is downregulated early and slightly up-regulated later in the time course. 

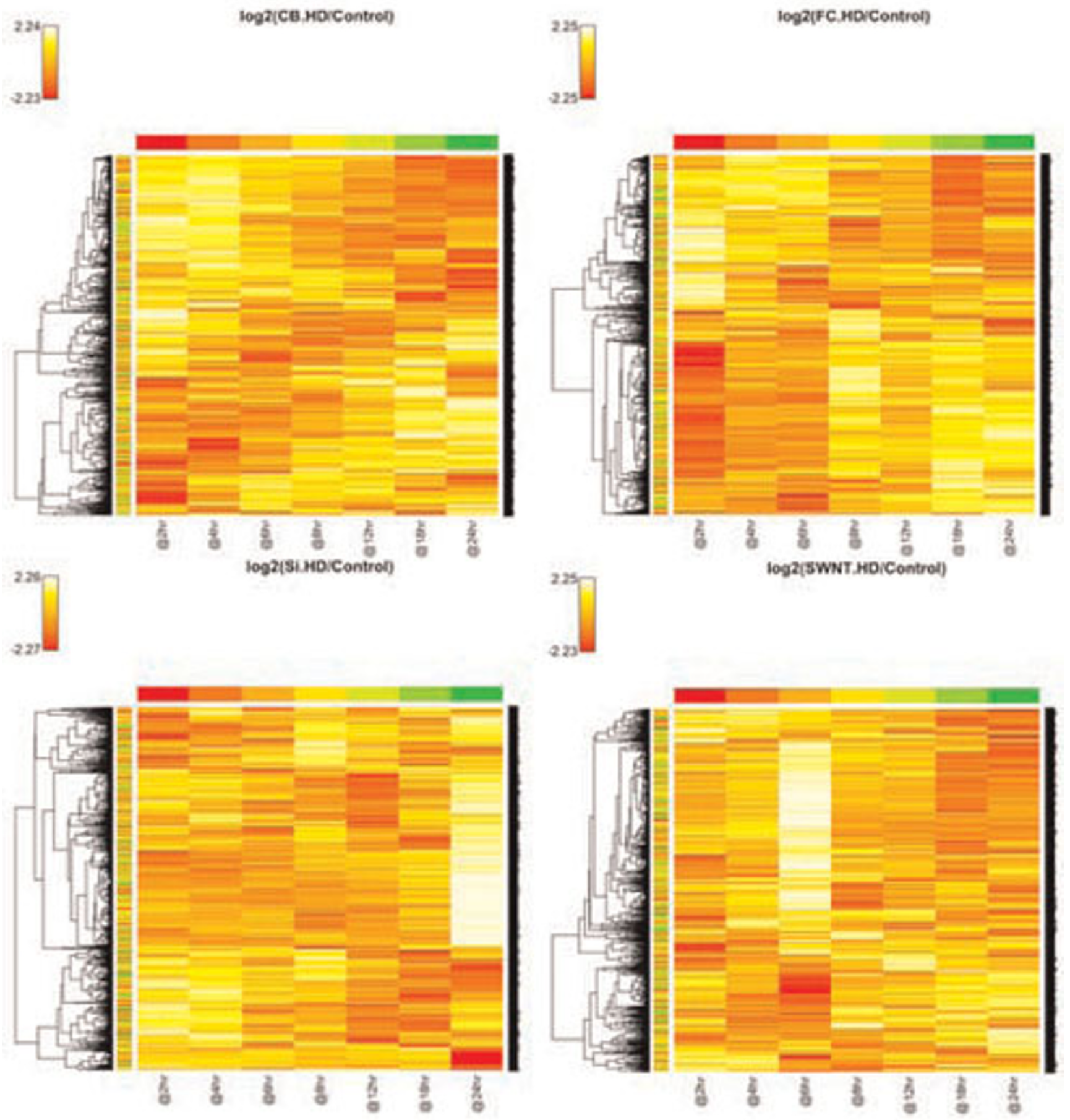

Figure I

Heat map and Hierarchical Clustering for the high-dose treatments.

\section{B. Multi-Dimensional Scaling (MDS) plots}

For the MDS plots, we consider the log of the absolute gene expression for each of the treatments and the baseline (no ratio is taken). There are 7 plots, one for each time point, which are displayed in Figures 3 and 4.
In these plots, the closer two points are to each other, the more similar their gene expression profiles are, according to the Pearson correlation (see Methods section). Considering, for instance, the plot at time 18 hrs, it can be easily seen that most of the 
228
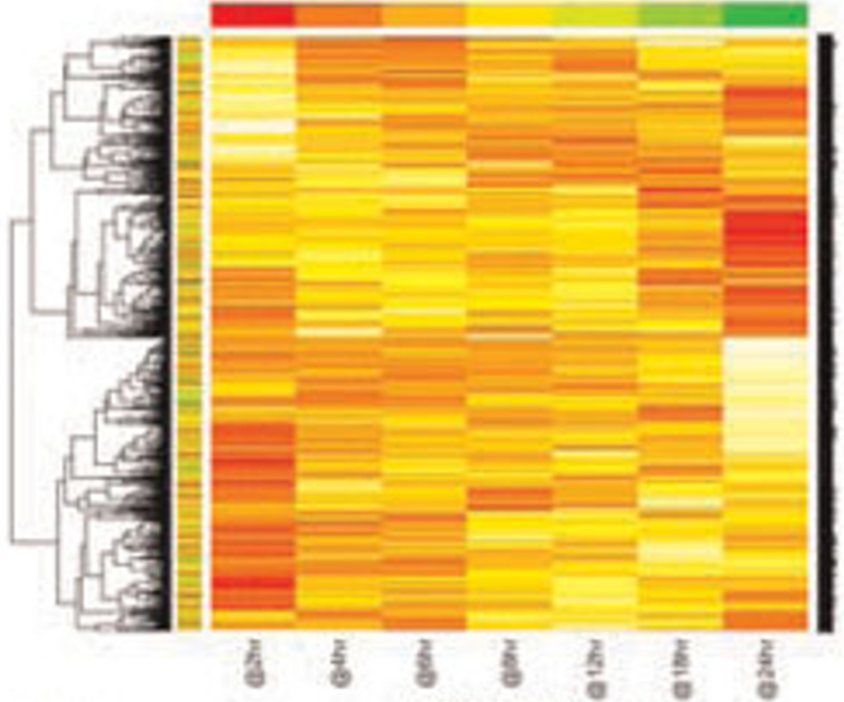

$\log 2$ (sicicontrob)
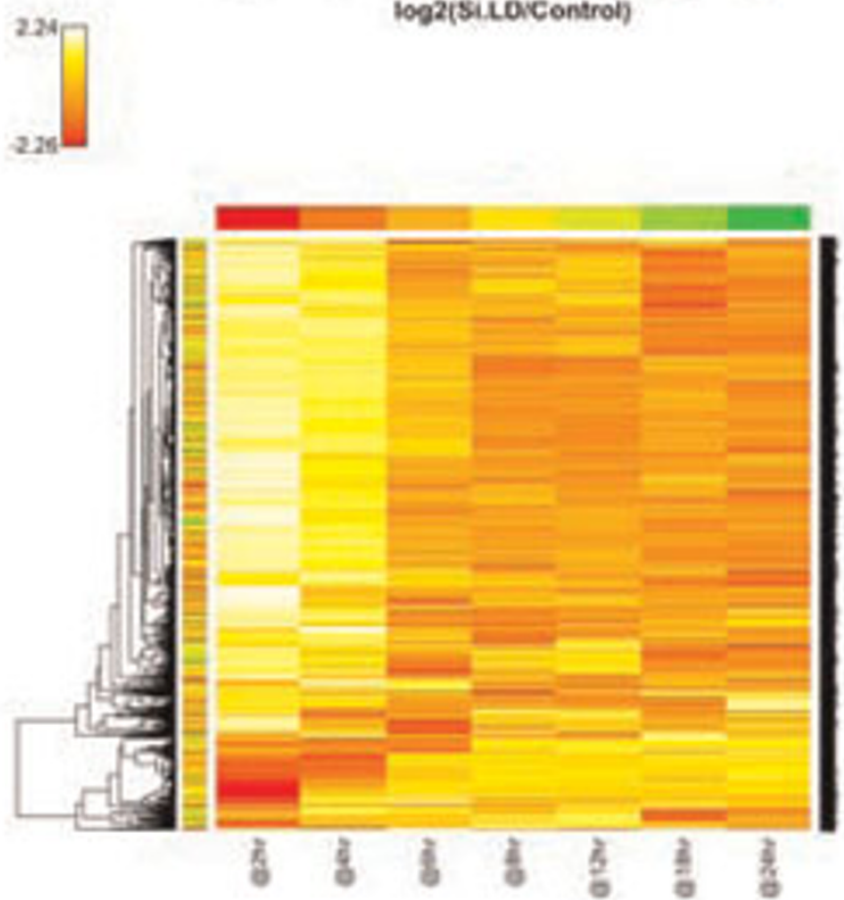

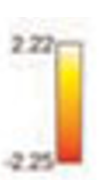

$\log 2$ (FC.LOV.Contron)

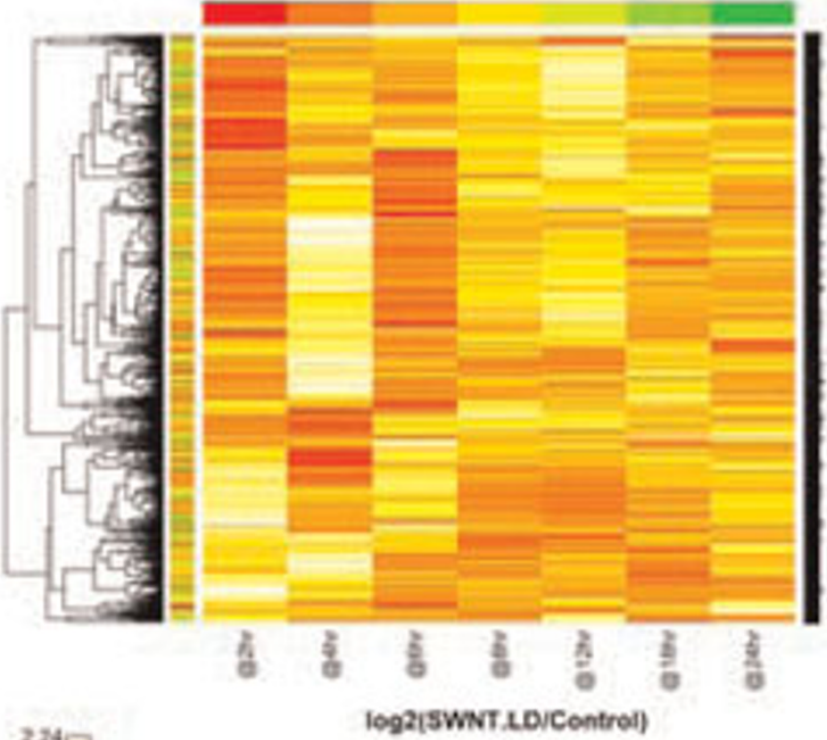

${ }_{223}^{224}$
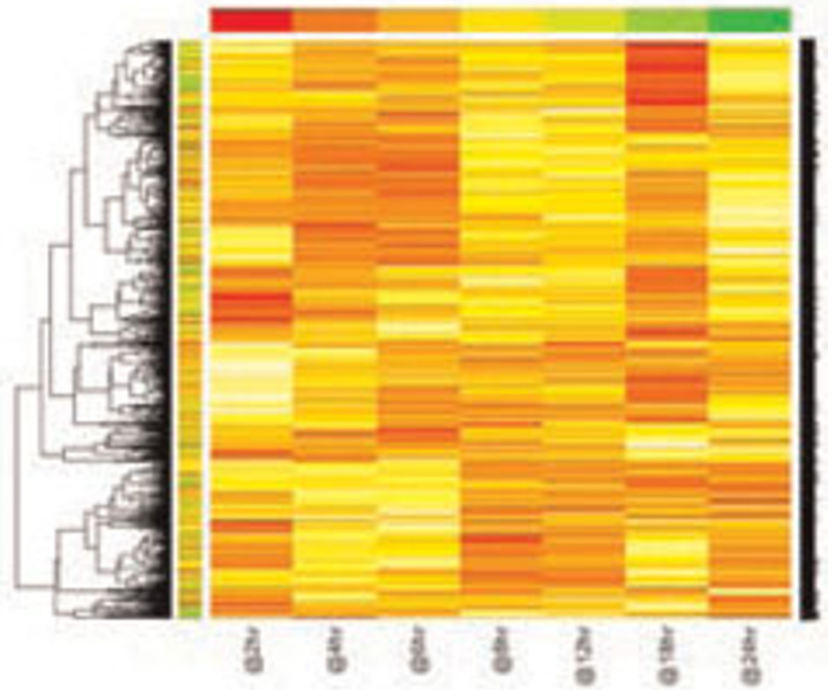

Figure 2

Heat map and Hierarchical Clustering for the low-dose treatments.

treatments show similar gene expression to the baseline at this time (i.e., a weak transcriptional response), while the $\mathrm{CB}$ HD (high dose) and FC HD treatments present a stronger response. This is clear by the fact that all the treatments are concentrated in one point except $\mathrm{CB}$ HD and FC HD. In fact, one observes that all treatments tend to become closer to the baseline as time proceeds, except for CB HD and FC HD. This shows that these two treatments, generally, have the most effect on changing gene expression. The same 

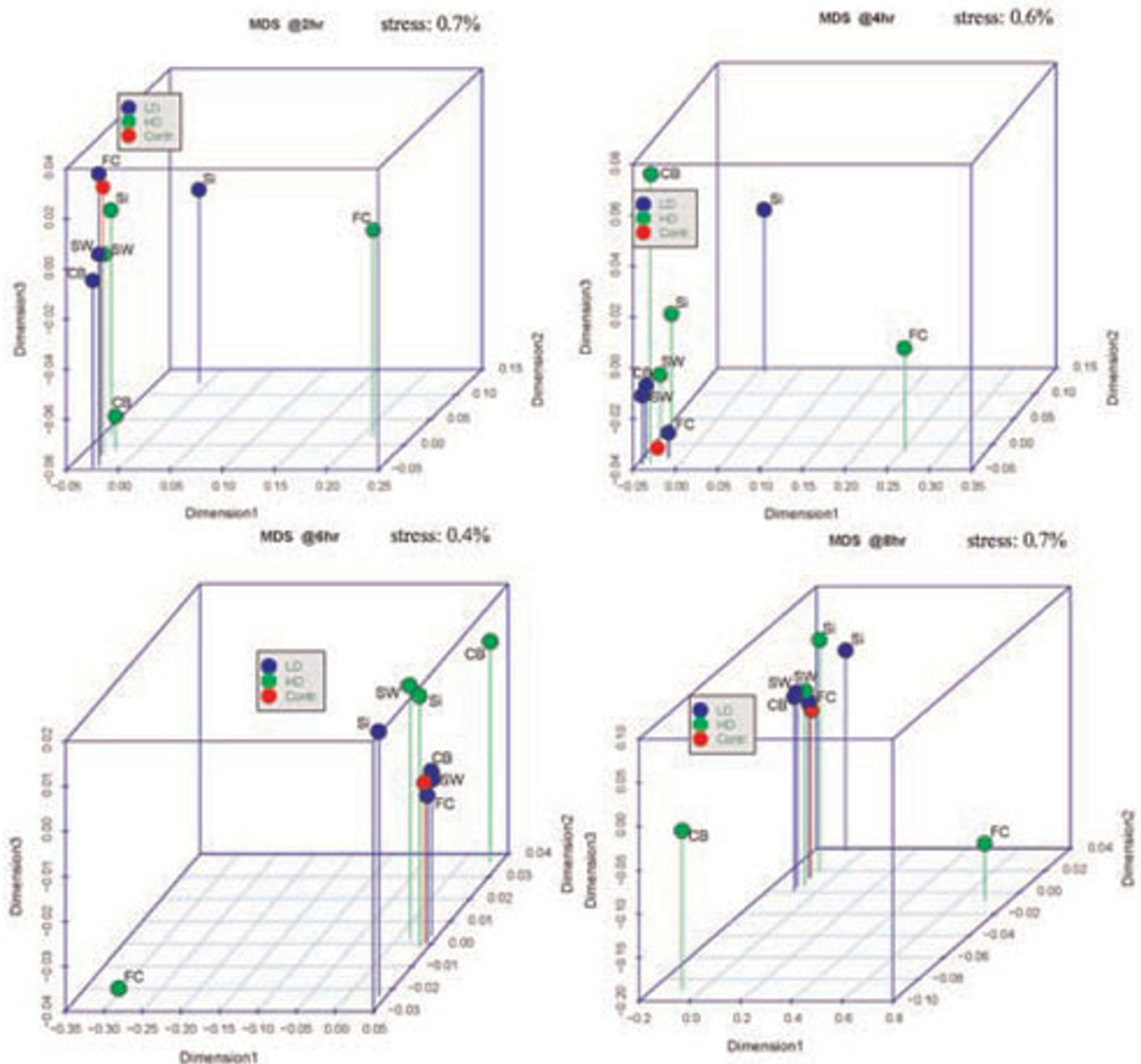

Figure 3

Sequence of MDS plot from time $2 \mathrm{hr}$ to $8 \mathrm{hr}$.

fact can also be observed in the heatmaps of Figures 1 and 2 .

\section{Self-Organizing Maps (SOM)}

Here, we again consider the log ratio between the gene expression in one of the treatment time-courses and the corresponding gene expression in the baseline timecourse, for each gene and each time point. Table 1 shows the optimal number of clusters for each treatment that were obtained by using a combination of the silhouette and CLEST criteria, as described in the Methods section. It is interesting to compare these numbers with what is deduced from visualizing the heatmaps in Figures 1 and 2. For example, Table 1 says that the number of clusters in $\mathrm{SiO}_{2} \mathrm{LD}$ and CB HD is 2 and 4, respectively. This fact is consistent with what one may infer from Figures 1 and 2.

Figure 5 shows the average expression values of genes in each cluster by performing SOM on the CB HD 

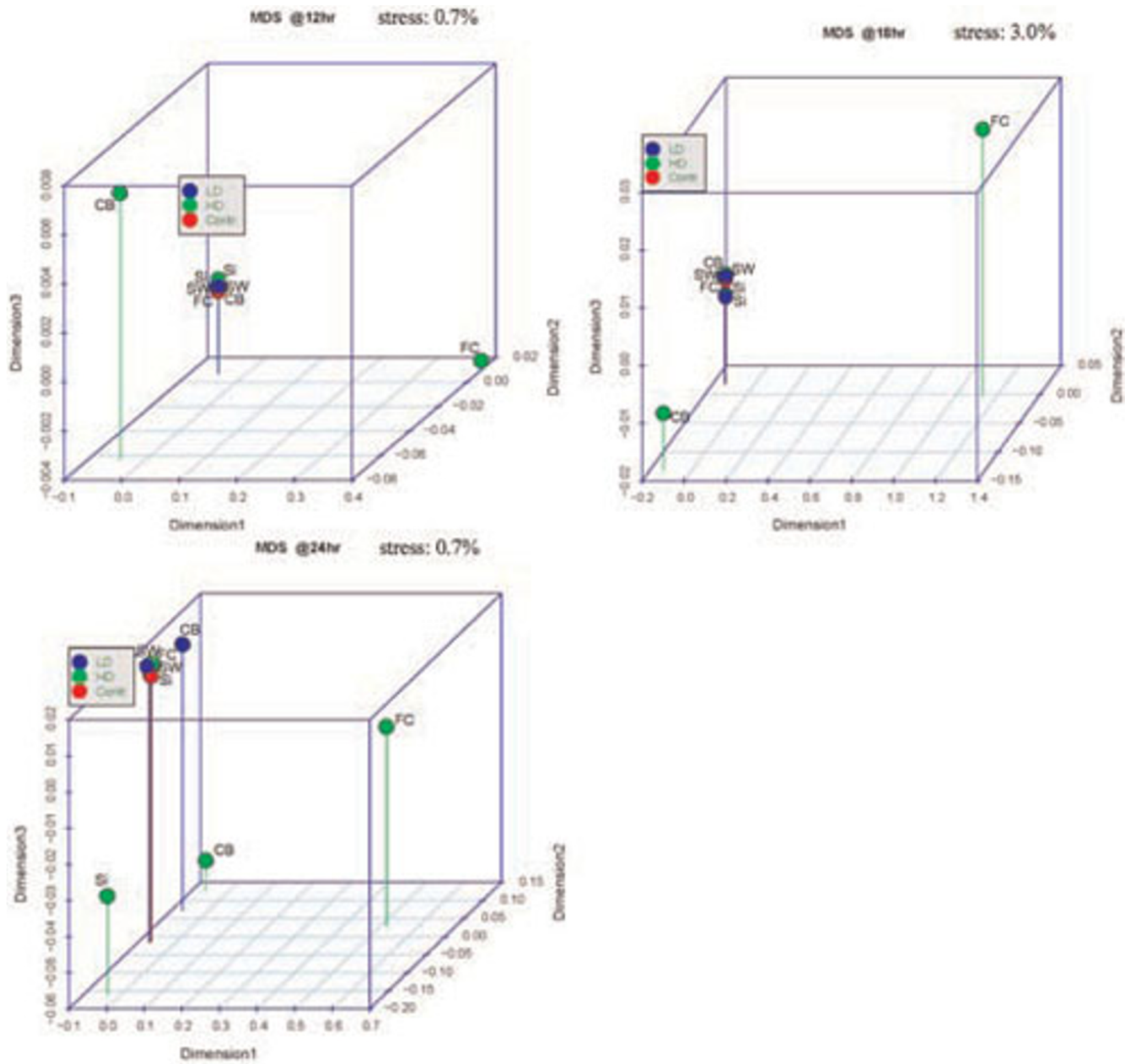

\section{Figure 4}

Sequence of MDS plot from time $12 \mathrm{hr}$ to $24 \mathrm{hr}$.

treatment (the plots for the other treatments, along with the list of genes in each cluster, can be found in the supplementary information). In agreement with the fact that the optimal number of clusters in this treatment was determined to be four, we employed $2 \times 2$ SOM to find four clusters. This can be contrasted with Figure 6, which shows the result for the same treatment but now using $5 \times$ 6 SOM to find 30 clusters. Obviously, as there are fewer genes in each cluster, we obtain tighter gene expression patterns (smaller standard deviations) in each cluster.

\section{ANOVA model}

The two-way ANOVA model that we consider has as factors time and dose, where the former has 3 levels (baseline, low dose and high dose) and the latter has 7 levels, corresponding to the seven time points in the experimental design. Since we are interested in the dose main effect on a given gene, we attempt to remove interaction of dose with time. After determining those genes affected significantly by the dose main effect, it is worthy to investigate between which specific levels there 
Table I: Treatments and the their determined optimal number of clusters

\begin{tabular}{lc}
\hline Treatment & Number of Clusters \\
\hline CB HD & 4 \\
CB LD & 5 \\
FC HD & 2 \\
FC LD & 3 \\
Sio2 HD & 10 \\
Sio2 LD & 2 \\
SWNT HD & 3 \\
SWNT LD & 5 \\
\hline
\end{tabular}

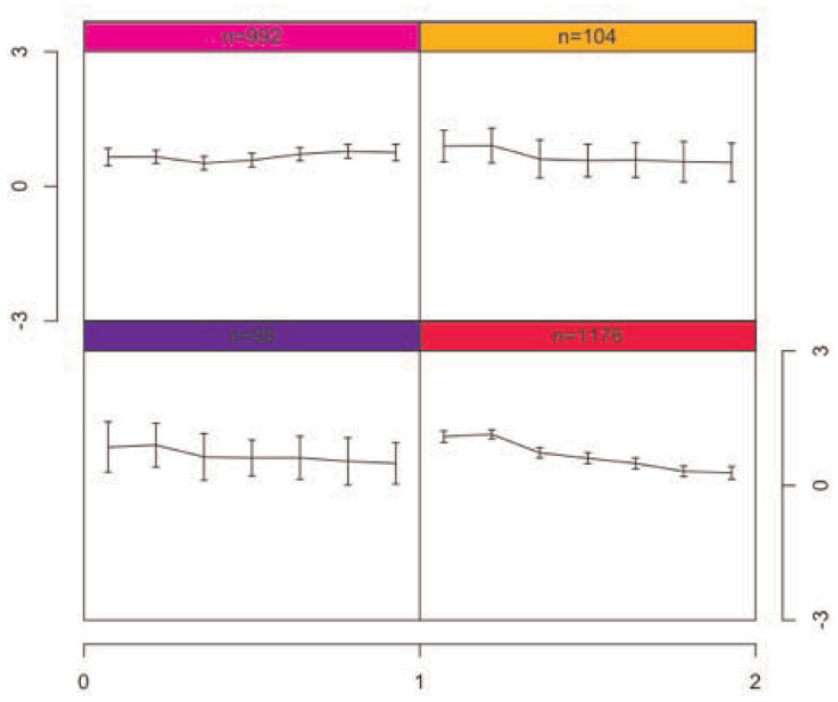

Figure 5

SOM clusters for a $2 \times 2$ unit plane for CB HD.

is a difference, which is accomplished by the Tukey's HSD post-hoc test (see the Methods section for a detailed description of the procedure that was employed).

Table 2 show the top 40 gene IDs that respond to the $\mathrm{CB}$ HD treatment, out of the original list of 2370 genes (the tables for the other treatments can be found in the supplementary information). The genes were ranked in ascending order of the $p$-value resulting from the ANOVA analysis, which means that the genes at the top are expected to show stronger responses to the treatment. In addition, the last three columns of Table 2 show where the expression differences lie, according to Tukey's HSD test; "1" indicates a significant difference.

\section{Discussion}

We comment here on the results obtained for the various treatments. The analysis resulted in several gene families or genes associated with the same cellular processes which responded significantly to the treatments. Very little toxicity overall was seen with any nanomaterial at the noncytotoxic dose except for silica, the positive control substance. Pathways for inflammation and irritation were identified; e.g., these pathways were observed for the silica at a noncytotoxic dose. At the cytotoxic doses, the most toxicity was seen with carbonyl iron - the explanation for this being the iron overload experienced by the cells. On the other hand, the majority of the genes found show significant differences at both the low and high dose levels. We observed that gene expression level becomes closer to basal level in later time points. This trend is seen with all gene expression profiling where the initial hours show the most activity $[49,50]$.

Next we provide next detailed comments on the genes displayed in Table 2, which are associated with significant response to the $\mathrm{CB}$ treatment. Many of these genes are notable. Three different chromodomain-helicase-DNA binding protein genes are seen: CHD2, CHD3 and CHD4. These genes code for proteins which form a histone deacetylase complex involved in chromatin remodeling, an important transcriptional process [22]. Two different genes coding for proteins in the protein tyrosine phosphatase or protein tyrosine phosphataselike families, PTP1B and PTPLB, are found to be highly significant. In addition, four genes associated with apoptosis are found. IL1A and TRADD are both involved in the NF-KappaB signaling pathways $[23,24]$. NR4A1 is an early response gene which codes for a nuclear orphan receptor and has an active role in cellular stress [25]. TAF9 is a TATA box-binding protein associated factor binds histones and is important in transcriptional regulation and cell viability [23-26]. Additionally, another protease, PSME4, which is related to the ubiquitin pathway, was found [27]. STX1A is a gene that may be involved Parkinson's disease as it translates to proteases located in brain tissue [28]. The gene PAIP1 is involved in the regulation of translation. PAIP1 is very similar to EIF4A (an eukaryotic initiation factor) which has a role in binding mRNA molecules to the ribosome. PAIP1 is active in mRNA turnover and their deadenylation and decay [29]. One gene, FRAG1, appears twice as two different FRAG1 probes were found to be significant within the top 40 genes.

Genes that show differential expression at both dose levels include MAPK8IP3, TRIO, ATP6V1F, and Hs. 656094 (gene index 2145). TRIO regulates the reorganization of the cytoskeleton and is essential for cell migration and cell growth [30]. ATP6V1F is active in transport mechanisms; it mediates the acidification of intracellular compartments essential to ion transport across cellular membranes [31,32]. MAPK8IP3 is 


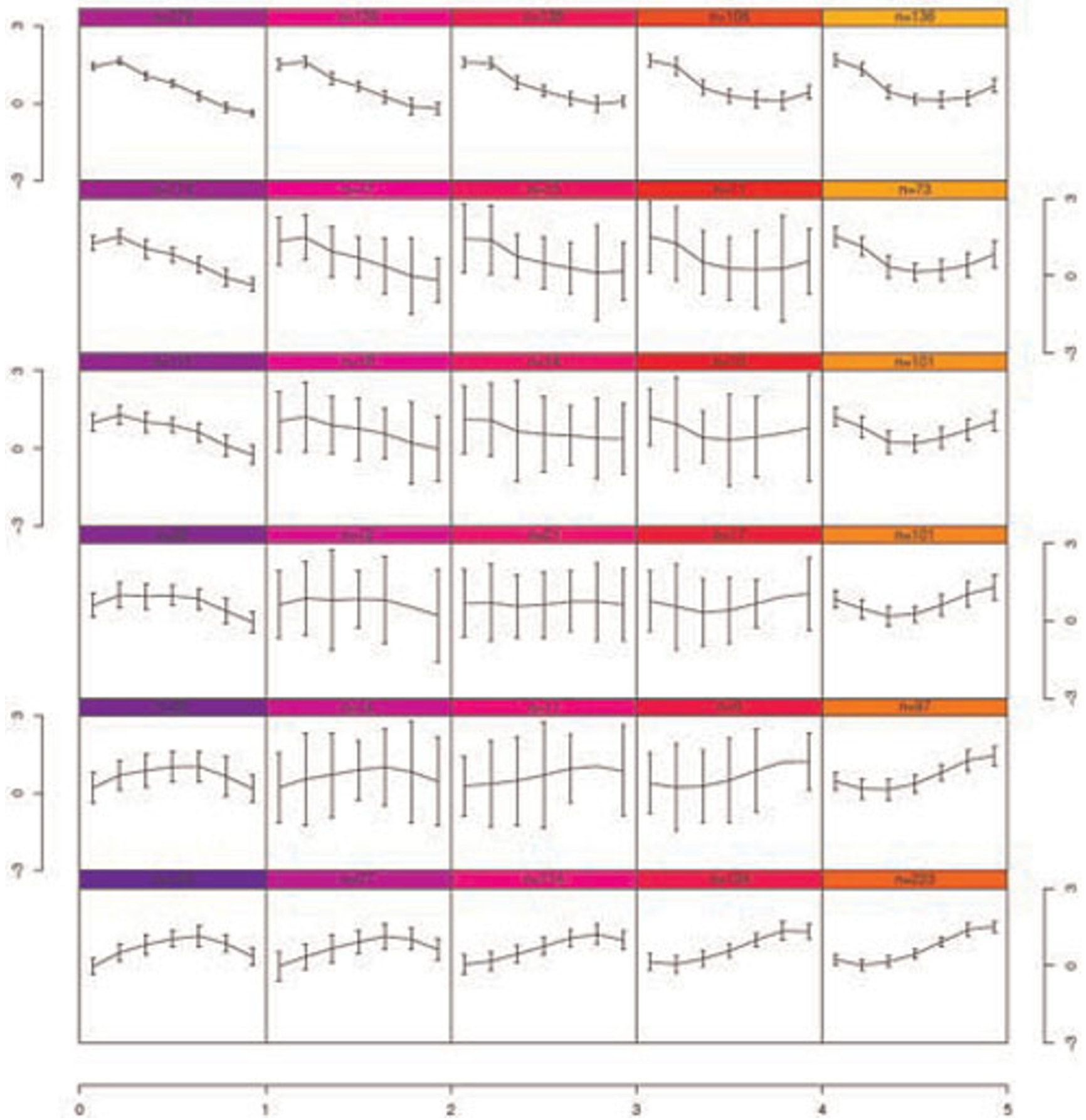

Figure 6

SOM clusters for a $5 \times 6$ unit plane for CB HD.

involved in signal transduction by coding for a scaffold protein in the JNK signaling pathway [33]. It is activated by cytokines and environmental stresses. One gene (Hs. 656094, gene index 2145) is unannotated, being a transcribed locus that translates to a protein with no known functions.
Several genes show differential expression only with the high dose. These genes include IL1A, LAMB2, IMPA1, ARID5B, CHD4 and ARHGAP22. The genes ARHGAP22, ARID5B and CHD4 are involved in the regulation of transcription. ARHGAP22 codes for a rac GPTase active in signal transduction and seems to play an indirect role 
Table 2: Genes showing significant difference among various level of dose in treatment CB

\begin{tabular}{|c|c|c|c|c|c|c|}
\hline Rank & Acc No. & Entrez Gene ID & Gene Name & HD-LD & HD-Control & LD-Control \\
\hline I & $A B 023163$ & 23390 & ZDHHCI7 & I & I & I \\
\hline 2 & NM_033495 & 90293 & KLHLI 3 & 1 & 1 & I \\
\hline 3 & AL3902I4 & 7204 & TRIO & I & I & 1 \\
\hline 4 & BF057080 & - & ENPPI & 1 & I & 1 \\
\hline 5 & NM_002292 & 3913 & LAMB2 & I & 1 & 0 \\
\hline 6 & NM_00423I & 9296 & ATP6VIF & I & I & 1 \\
\hline 7 & NM_004524 & 3993 & LLGL2 & I & I & I \\
\hline 8 & U9I543 & 1107 & CHD3 & I & I & I \\
\hline 9 & AF052I59 & 201562 & PTPLB & 1 & 1 & 0 \\
\hline 10 & AL04947I & - & ARID5B & 1 & 1 & 0 \\
\hline II & NM_00I46I & 2330 & FMO5 & I & I & I \\
\hline 12 & NM_002I35 & 3164 & NR4AI & I & I & 0 \\
\hline 13 & NM_002827 & 5770 & PTPIB & I & I & 1 \\
\hline 14 & NM_0045I7 & 3611 & ILK & 0 & 1 & 1 \\
\hline 15 & NM_0I4489 & 27315 & FRAGI & 1 & I & I \\
\hline 16 & AL0492I8 & - & RNASEH2B & I & I & I \\
\hline 17 & BC0I3306 & 6923 & TCEB2 & I & I & I \\
\hline 18 & D3852I & 23198 & PSME4 & I & I & I \\
\hline 19 & NM_00I 273 & 1108 & CHD4 & 1 & 1 & 0 \\
\hline 20 & NM_003789 & 8717 & TRADD & I & I & I \\
\hline 21 & NM_005374 & 4355 & MPP2 & 0 & 1 & I \\
\hline 22 & NM_007| I 2 & 7059 & THBS3 & 1 & 1 & 1 \\
\hline 23 & AF090094 & - & OAZI & 0 & I & I \\
\hline 24 & NM_000575 & 3552 & ILIA & I & I & I \\
\hline 25 & NM_003 |87 & 6880 & TAF9 & 1 & I & I \\
\hline 26 & NM_005309 & 2875 & GPT & 1 & 1 & 1 \\
\hline 27 & NM_00645 I & 10605 & PAIPI & 1 & I & 0 \\
\hline 28 & NM_022365 & 64215 & DNAJCI & I & I & I \\
\hline 29 & Al435998 & - & Cl7orf5I & 1 & 1 & 1 \\
\hline 30 & AW02I63I & - & & I & I & I \\
\hline 31 & NM_004603 & 6804 & STXIA & 1 & 1 & 0 \\
\hline 32 & NM_005I82 & 766 & CA7 & 1 & 1 & 1 \\
\hline 33 & AA60I902 & - & MAPK8IP3 & 1 & I & I \\
\hline 34 & ALI 37484 & 221191 & KLKBL4 & 1 & 1 & 1 \\
\hline 35 & NM_00I27I & 1106 & CHD2 & 1 & I & 1 \\
\hline 36 & NM_005536 & 3612 & IMPAI & 1 & 1 & 0 \\
\hline 37 & X58529 & 3507 & IGHM & 0 & 1 & I \\
\hline 38 & NM_0I4489 & 27315 & FRAGI & 1 & 1 & I \\
\hline 39 & NM_0I6574 & 1813 & DRD2 & 1 & 1 & 1 \\
\hline 40 & NM_023 III I & 2260 & FGFRI & I & I & I \\
\hline
\end{tabular}

in cytoskeletal reorganization and cell motility [34]. ARID5B regulates transcription by binding to DNA and has important roles in development and differentiation [35]. CHD4 represses transcription by modulating the amount of protein at the centrosome during mitosis [36]. IL1A is involved in apoptosis as well as many roles in immune and inflammatory responses and hematopoiesis [37]. The last two genes, LAMB2 and IMPA1 are both involved in development. IMPA1 has an essential role in phosphatidylinositol pathways and LAMB2 codes for a laminin isoform which is involved in embryonic development and the organization of cells into tissues [38].

Table 3 breaks down where differences are found among the 40 genes on the top of list of each treatment. It can be seen from this table that $\mathrm{CB}$ is a treatment under which there appear to be stark differences in gene expression between all dose levels. On the other hand, most of the response due to exposure to FC occurs under a high dose level, the differences between low dose and no treatment having a much smaller representation. For $\mathrm{SiO}_{2}$ and SWNT, most of the difference is between high or low dose (it does not matter) and no treatment. These observations are consistent with what was inferred from the heatmaps and MDS plots in Figures 1, 2, 3, and 4.

Table 3: Number of genes that have significant differences among the top $\mathbf{4 0}$ genes

\begin{tabular}{llll}
\hline & HD-LD & HD-Cont. & LD-Cont. \\
\hline CB & 36 & 40 & 32 \\
FC & 40 & 40 & 7 \\
SiO2 & 25 & 40 & 40 \\
SWNT & 27 & 40 & 39 \\
\hline
\end{tabular}




\section{Conclusion}

The analysis presented here lead to interesting and complementary conclusions about the response across time of human epidermal keratinocytes after exposure to nanomaterials. For example, we observed (especially through the time sequence of MDS plots) that gene expression for each treatment become closer to the expression of the baseline cultures as time proceeds, except for $\mathrm{CB} \mathrm{HD}$ and FC HD, which generally have the most changing expression levels. The results from the MDS analysis mostly confirmed the results from the heatmaps and SOM clustering. The genes found to be differentially-expressed by the ANOVA analysis are involved in a number of cellular processes, including regulation of transcription and translation, protein localization, transport, cell cycle progression, cell migration, cytoskeletal reorganization, signal transduction, and development. The majority of these genes show significant differences at both the low and high dose levels.

\section{Supplementary information}

http://www.ece.tamu.edu/ ulisses/HARC/index.html

\section{Competing interests}

The authors declare that they have no competing interests.

\section{Authors' contributions}

AZ: Bioinformatics, drafted the manuscript; For MJC: cell culture, coordinated RNA isolation and microarray processing, conceived the study, helped draft the manuscript; UBN: Bioinformatics, helped draft the manuscript; ERD: Participated in design and coordination and helped draft the manuscript.

\section{Acknowledgements}

This work was funded by the Houston Advanced Research Center (HARC) and by the National Science Foundation (NSF), through NSF awards BES-0536679 (Cunningham), CCF-0845407 (Braga-Neto), and CCF-0634794 (Dougherty).

This article has been published as part of BMC Bioinformatics Volume 10 Supplement II, 2009: Proceedings of the Sixth Annual MCBIOS Conference. Transformational Bioinformatics: Delivering Value from Genomes. The full contents of the supplement are available online at http://www.biomedcentral.com/I47I-2/05/I0? issue=SII.

\section{References}

I. Roco MS, Bainbridge WS and eds: Societal Implications of Nanoscience and Nanotechnology. National Science Foundation, NSET Workshop Report Kluwer Academic Publishers, Norwell, MA; 2001.

2. Jortner J and Rao CNR: Nanostructured advanced materials. Perspectives and directions. Pure Appl Chem 2002, 74: $1491-1506$.

3. Oberdorster G, Oberdorster E and Oberdorster J: Nanotoxicology: an emerging discipline evolving from studies of ultrafine particles. Environ Health Perspect 2005, I I 3:823-839.
4. Lam C, James JT, McCluskey R and Hunter RL: Pulmonary toxicity of single-wall carbon nanotubes in mice $\mathbf{7}$ and $\mathbf{9 0}$ days after intratracheal instillation. Toxicol Sci 2004, 77: I26-I34.

5. Warheit DB, Laurence BR, Reed KL, Roach DH, Reynolds GAM and Webb TR: Comparative pulmonary toxicity assessment of single wall carbon nanotubes in rats. Toxicol Sci 2004, 77:II7-125.

6. Monteiro-Riviere NA and Inman AO: Challenges for assessing carbon nanomateral toxicity to the skin. Carbon 2006, 44:1070-1078.

7. Nuwaysir EF, Bittner M, Trent J, Barrett JC and Afshari CA: Microarrays and toxicology: the advent of toxicogenomics. Mol Carcinog 1999, 24:153-159.

8. Cunningham MJ and Shah M: Toxicogenomics. "Handbook of Pharmaceutical Biotechnology" John Wiley \& Sons, Inc., NJ: Gad SC 2007, 229-25I.

9. Cunningham MJ: Genomics and proteomics: the new millennium of drug discovery and development. J Pharmacol Toxicol Methods 2000, 44:29I-300.

10. Braxton $\mathrm{S}$ and Bedilion $\mathrm{T}$ : The integration of microarray information in the drug development process. Curr Opin Biotechnol 1998, 9:643-649.

II. Ulrich $\mathrm{R}$ and Friend $\mathrm{SH}$ : Toxicogenomics and drug discovery: will new technologies help us produce better drugs?. Nat Rev Drug Discov 2002, I:84-88.

12. Brun YF, Varma R, Hector SM, Pendyala L, Tummala R and Greco WR: Simultaneous modeling of concentration-effect and time-course patterns in gene expression data from microarrays. Cancer Genomics Proteomics 2008, 5(I):43-53.

13. Cunningham MJ, Magnuson SR and Falduto MT: Gene expression profiling of nanoscale materials using a systems biology approach. Toxicol Sci 2005, 84(S-I):9.

14. Shah M, Lema $C$ and Cunningham MJ: In vitro studies of singlewalled carbon nanotubes: a toxicogenomics approach. Toxicol Sci 2007, 96(S-I): I 33.

15. Cunningham MJ: Gene-Cellular Interactions of Nanomaterials: Genotoxicity to Genomics. "Nanotoxicology: Characteristics, Dosing and Health Effects on Target Organs" Taylor \& Francis/Informa Healthcare, New York, New York: Monteiro-Riviere N, Tran L 2007, I73-196.

16. Alizadeh AA, Eisen MB, Davis RE, Ma C, Lossos IS, Rosenwald A Boldrick JC, Sablet H, Tran T, Yu X, Powell JI, Yamng L, Marti GE, Moore T, Hudson J, Lu L, Lewis DB, Tibshirani R, Sherlock G, Chan WC, Greiner TC, Welsenburger DD, Armitage JO, Warnke R, Levy R, Wilson W, Grever MR, Boyd JC, Botstein D, Brown PO and Staudt LM: Distinct types of diffuse large B-cell lymphoma identified by gene expression profiling. Nature 2000, 403:503-5II.

17. Tamayo P, Slonim D, Mesirov J, Zhu Q, Kitareewan S, Dmitrovsky E, Lander ES and Golub TR: Interpreting patterns of gene expression with self-organizing maps: Methods and application to hematopoietic differentiation. Genetics 1999, 96:2907-2912.

18. Kaufman L and Rousseeuw PJ: Finding Groups in Data - An Introduction to Cluster Analysis. Wiley Series in Probability and Mathematical Statistics; 1990.

19. Dudoit $S$ and Fridyland J: A prediction-based resampling method for estimating the number of clusters in a dataset. Genome Biol 2002, 3:RESEARCH 0036.I-0036.2I.

20. Neter J, Kutner MH, Nachtsheim CJ and Wasserman W: Applied Linear Statistical Models.McGraw-Hill/Irwin; 41996.

21. Hardle W and Simar L: Applied Multivariate Statistical Analysis.Springer; 22007.

22. Woodage Trevor, Basrai A Munira, Baxevanis D Andreas, Hieter Philip and Collins S Francis: Characterization of the CHD family of proteins. Proc Natl Acad Sci USA I 997, 94(2I): | |472-I| 477.

23. Soloff S Melvyn, Izban G Michael, Cook L Dennis Jr, Jeng Yow-Jiun and Mifflin C Randy: Interleukin-I-induced NF-\{kappa\}B recruitment to the oxytocin receptor gene inhibits RNA polymerase II-promoter interactions in cultured human myometrial cells. Mol Hum Reprod 2006, I 2:6I9-624.

24. Bock Walter Karl and Köhle Christoph: Ah receptor- and TCDDmediated liver tumor promotion: clonal selection and expansion of cells evading growth arrest and apoptosis. Biochem Pharmacol 2003, 66: I 403-I408.

25. Maxwell $M$ and Muscat G: The NR4A subgroup: immediate early response genes with pleiotropic physiological roles. Nucl Recept Signal 4:e002. 
26. Frontini Mattia, Soutoglou Evi, Argentini Manuela, BoleFeysot Christine, Jost Bernard, Scheer Elisabeth and Tora Làszlò: TAF9b (Formerly TAF9L) Is a Bona Fide TAF That Has Unique and Overlapping Roles with TAF9. Mol Cell Biol 2005, 25:4638-4649.

27. Khor Bernard, Bredemeyer L Andrea, Huang Ching-Yu, Turnbull R Isaiah, Evans Ryan, Maggi B Leonard Jr, White J Michael, Walker M Laura, Carnes Kay, Hess A Rex and Sleckman P Barry: Proteasome Activator PA200 Is Required for Normal Spermatogenesis. Mol Cell Biol 2006, 26:2999-3007.

28. Brose Nils: Altered Complexin Expression in Psychiatric and Neurological Disorders: Cause or Consequence? Mol Cells 2008, 25:7-19.

29. Craig WB Andrew, Haghighat Ashkan, Yu TK Annie and Sonenberg Nahum: Interaction of polyadenylate-binding protein with the elF4G homologue PAIP enhances Translation. Nature 1998, 392:520-523.

30. Seipel K, Medley QG, Kedersha NL, Zhang XA, O'Brien SP, SerraPages C, Hemler ME and Streuli M: Trio amino-terminal guanine nucleotide exchange factor domain expression promotes actin cytoskeleton reorganization, cell migration and anchorage-independent cell growth. J Cell Sci I999, I | 2:1825-1834.

31. Jacobsson A Josefin, Haitina Tatjana, Lindblom Jonas and Fredriksson Robert: Identification of six putative human transporters with structural similarity to the drug transporter SLC22 family. Genomics 2007, 90:595-609.

32. Imai-Senga Yoko, Sun-Wada Ge-Hong, Wada Yoh and Futai Masamitsu: A human gene, ATP6EI, encoding a testisspecific isoform of H+-ATPase subunit E. Gene 2002, 289:7-12.

33. Ito Michihiko, Yoshioka Katsuji, Akechi Mizuho, Yamashita Shinya, Takamatsu Nobuhiko, Sugiyama Kenji, Hibi Masahiko, Nakabeppu Yusaku, Shiba Tadayoshi and Yamamoto Ken-Ichi: JSAPI, a Novel Jun N-Terminal Protein Kinase (JNK)Binding Protein That Functions as a Scaffold Factor in the JNK Signaling Pathway. Mol Cell Biol 1999, 19:7539-7548.

34. Sanz-Moreno Victoria, Gadea Gilles, Ahn Jessica, Paterson Hugh, Marra Pierfrancesco, Pinner Sophie, Sahai Erik and Marshall j Christopher: Rac Activation and Inactivation Control Plasticity of Tumor Cell Movement. Cell 2008, I35:5I0-523.

35. Patsialou Antonia, Wilsker Deborah and Moran Elizabeth: DNAbinding properties of ARID family proteins. Nucleic Acids Res 2005, 33:66-80.

36. Hong Uk Kyung, Park Soo Young, Seong Yeon-Sun, Kang Dongmin, Bae Chang-Dae and Park Joobae: Functional Importance of the Anaphase-Promoting Complex-CdhI-Mediated Degradation of TMAP/CKAP2 in Regulation of Spindle Function and Cytokinesis. Mol Cell Biol 2007, 27:3667-368I.

37. Hogquist KA, Nett MA, Unanue ER and Chaplin DD: Interleukin I is processed and released during apoptosis. PNAS I991, 88:8485-9.

38. Tzu Julia and Marinkovich M Peter: Bridging structure with function: Structural, regulatory, and developmental role of laminins. Int J Biochem Cell Biol 2008, 40:199-2/4.

39. Cunningham MJ, Liang S, Fuhrman S, Seilhamer JJ and Somogyi R: Gene expression microarray data analysis for toxicology profiling. Annals of the New York Academy of Sciences 2000, 919:52-67.

40. Cunningham MJ, Zweiger G, Bailey D, Schwartz M, Braxton S and Seilhamer J: Application of a genome-based screen. Archives of Pharmacology 1998, 358(I, Suppl 2):R564.
Publish with Bio Med Central and every scientist can read your work free of charge

"BioMed Central will be the most significant development for disseminating the results of biomedical research in our lifetime. "

Sir Paul Nurse, Cancer Research UK

Your research papers will be:

- available free of charge to the entire biomedical community

- peer reviewed and published immediately upon acceptance

- cited in PubMed and archived on PubMed Central

- yours - you keep the copyright
BioMedcentral 